\section{Underutilization of Aldosterone Antagonists in Heart Failure}

\author{
Shanise J. Patterson', Anne B. Reaves ${ }^{1,2}$, Elizabeth A. Tolley², \\ Dagny Ulrich ${ }^{3}$, Christopher Hilty', Catherine J. Clarke ${ }^{1,2}$, and \\ Timothy H. Self ${ }^{2}$
}

Hospital Pharmacy

2017, Vol. 52(10) 698-703

(c) The Author(s) 2017

Reprints and permissions:

sagepub.com/journalsPermissions.nav

DOI: $10.1177 / 0018578717724886$

journals.sagepub.com/home/hpx

@SAGE

\begin{abstract}
Background: Treatment with an aldosterone antagonist (AA) has been shown in multiple trials to reduce heart failure (HF)related morbidity, mortality, and hospital readmission. American College of Cardiology Foundation (ACCF) and American Heart Association (AHA) treatment guidelines recommend the use of an AA in all HF patients with an ejection fraction $\leq 35 \%$ and no known contraindication. Several studies have documented underuse of AA. Objectives: To determine the proportion of patients who received AA therapy consistent with the ACCF/AHA guidelines. Secondary objectives included determining the proportion of patients who received an AA inconsistent with guidelines and 30- and 90-day readmission rates. Methods: A retrospective chart review was conducted of patients admitted to an inner city academic medical center with a diagnosis of HF between August 16, 20II, and June 5, 2013. Results: A total of $346 \mathrm{HF}$ admissions (87.6\% African American) were evaluated. Use of an AA at discharge was consistent with guidelines in $31 \%$ of patients. A total of $I 2 I$ patients (35\%) were discharged on an AA. Among the remaining 225 patients who were not discharged on an AA, $170(75.6 \%)$ had no contraindication to therapy. Sixty-one patients were readmitted within 30 days, and a total of 108 patients were readmitted within 90 days. There were no significant differences in readmission rates between patients who were discharged on AA therapy and those who were not. Conclusion: AAs are still underutilized in the treatment of HF.
\end{abstract}

\title{
Keywords
}

aldosterone antagonists, heart failure, underuse

\section{Introduction}

Aldosterone antagonists reduce the risk of mortality, morbidity, and readmission rates among patients with heart failure (HF). The Randomized Aldactone Evaluation Study (RALES) found that patients who received spironolactone in combination with angiotensin-converting enzyme (ACE) inhibitors and loop diuretics had a 30\% reduction in risk of death from progressive HF and sudden death from cardiovascular causes in patients with New York Heart Association (NYHA) class III and IV HF. ${ }^{1}$ Further studies, EPHESUS and EMPHASIS-HF, found significant reduction in mortality associated with the use of eplerenone in patients with postmyocardial infarction and NYHA class II HF, respectively. ${ }^{2,3}$ Recently, TOPCAT concluded that patients who had a preserved ejection fraction (EF) and received spironolactone had a reduction in hospitalizations but no reduction in mortality. ${ }^{4}$ The American College of Cardiology Foundation (ACCF) and American Heart Association (AHA) have joint evidence-based treatment guidelines for the management of patients with HF, yet many do not receive optimal drug therapy. ${ }^{5-7}$

The ACCF/AHA guidelines recommend the use of an aldosterone antagonist in patients with NYHA class II to IV $\mathrm{HF}$ and an $\mathrm{EF}$ of $\leq 35 \%$, unless contraindicated (class I recommendation). ${ }^{2}$ Serum creatinine should be $\leq 2.5 \mathrm{mg} / \mathrm{dL}$ in men or $\leq 2.0 \mathrm{mg} / \mathrm{dL}$ in women (glomerular filtration rate [GFR], $>30 \mathrm{~mL} / \mathrm{min}$ ), and potassium should be $<5.0 \mathrm{mEq} / \mathrm{L}$. ${ }^{8}$

Albert et $\mathrm{al}^{6}$ observed 43625 patients admitted with the primary diagnosis of HF. Of the 12565 patients who met guideline criteria for an aldosterone antagonist, only 4087 received a prescription at discharge, which is less than onethird of patients eligible for therapy. The investigators also assessed for inappropriate use of aldosterone antagonist therapy and found that only $3.12 \%$ of patients who received therapy had a documented contraindication.

Curtis et $\mathrm{al}^{7}$ assessed adherence to HF treatment guidelines in a Medicare population transitioning from the hospital to an outpatient setting. A total of 2086 patients met criteria for an aldosterone antagonist, but only 561 (26.9\%) patients were prescribed one at discharge. Within 90 days of

\footnotetext{
'Methodist University Hospital, USA

${ }^{2}$ The University of Tennessee Health Science Center, Memphis, USA

${ }^{3}$ St. Jude Children's Research Hospital, USA

Corresponding Author:

Timothy H. Self, The University of Tennessee Health Science Center, 88I Madison Avenue, Memphis, TN 38I63, USA.

Email: tself@uthsc.edu
} 


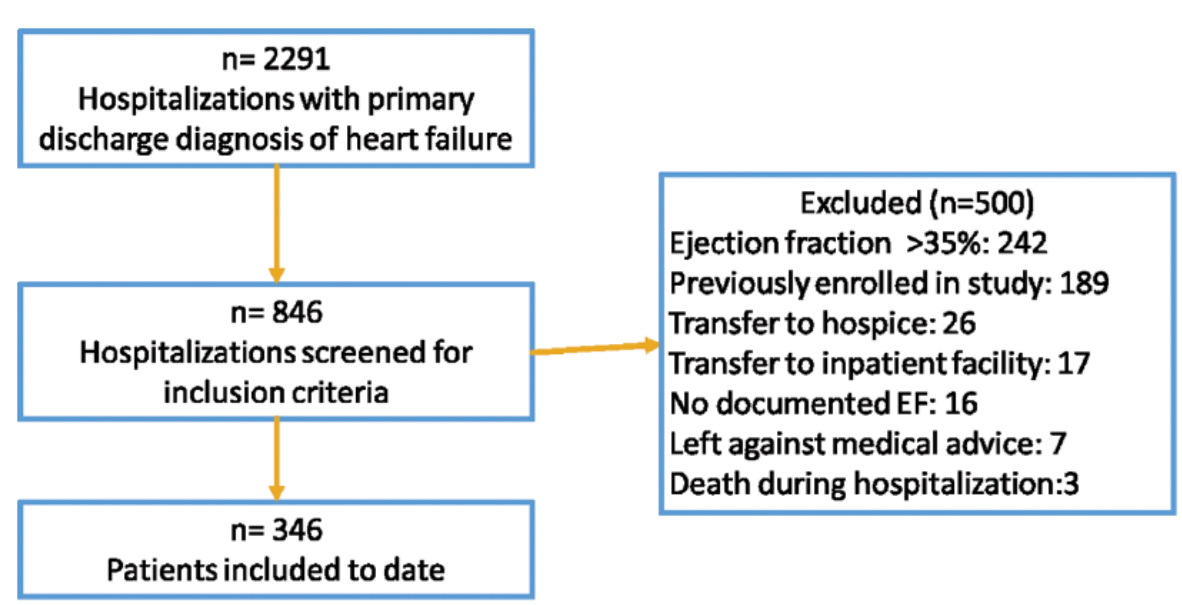

Figure I. Patient selection. Note. $\mathrm{EF}=$ ejection fraction.

discharge, $78.6 \%$ of the 561 patients discharged on therapy filled the prescription compared with $13 \%$ of eligible patients who were not given a discharge prescription. The study by Curtis et $\mathrm{al}^{7}$ supports previously published results: The majority of patients eligible for an aldosterone antagonist do not receive a prescription at discharge. In addition, they found that, if an aldosterone antagonist was not initiated during inpatient stay, it was less likely to be initiated after the patient left the hospital. The primary purpose of our study was to determine whether aldosterone antagonists are prescribed appropriately (only reduced EF) in our inner city, university-affiliated hospital.

\section{Methods}

A retrospective chart review of patients with a primary discharge diagnosis of HF between August 16, 2011, and June 5, 2013, was conducted at a 662-bed inner city hospital in an academic medical center. The study was approved by the affiliated university's institutional review board. Patients were enrolled in reverse chronological order if met inclusion criteria and received aldosterone antagonist therapy (Figure 1). Patients were identified using International Classification of Diseases, Ninth Revision (ICD-9) codes for HF to include: 428.0, 428.2, 428.20, 428.21, 428.23, 428.4, 428.40, 428.41, 428.42 and 428.9. Based on the ACCF/AHA treatment guidelines, the study included patients with a documented $\mathrm{EF} \leq 35 \%$ within 1 year prior to discharge and age greater than 18 years. To characterize the study population, data were collected from electronic medical record and included the following: age, sex, race, pertinent medical history, left ventricular EF, serum potassium at discharge, serum creatinine at discharge, estimated GFR at discharge, readmission status at 30 days and 90 days, and the presence of other therapies utilized in the treatment of HF (including ACE inhibitor, angiotensin receptor blocker [ARB], beta-blocker, isosorbide mononitrate/dinitrate, hydralazine, digoxin, loop diuretic). Patients were excluded if they left the hospital against medical advice, enrolled in hospice, transferred to another inpatient facility, were pregnant, or had a documented allergy to an aldosterone antagonist (spironolactone and/or eplerenone).

The primary objective was to determine the proportion of patients who received aldosterone antagonist therapy (spironolactone or eplerenone) consistent with the ACCF/AHA guidelines at discharge for the treatment of HF. Secondary objectives were to determine the proportion of patients who received aldosterone antagonist therapy inconsistent with the $\mathrm{ACCF} / \mathrm{AHA}$ guidelines and to compare readmission rates at 30 and 90 days.

The use of an aldosterone antagonist consistent with the ACCF/AHA guidelines at discharge was defined as use in an HF patient with $\mathrm{EF} \leq 35 \%$ and serum creatinine $<2.5 \mathrm{mg} / \mathrm{dL}$ for men, serum creatinine $<2 \mathrm{mg} / \mathrm{dL}$ for women and estimated GFR $>30 \mathrm{~mL} / \mathrm{min}$ and serum potassium $<5 \mathrm{mEq} / \mathrm{L}$ and avoidance in an HF patient who had renal dysfunction. The use of aldosterone antagonist inconsistent with the $\mathrm{ACCF} / \mathrm{AHA}$ guidelines at discharge was defined as use in an HF patient with serum creatinine $\geq 2.5 \mathrm{mg} / \mathrm{dL}$ for men, serum creatinine $\geq 2 \mathrm{mg} / \mathrm{dL}$ for women or estimated GFR $\leq 30 \mathrm{~mL} / \mathrm{min}$ or serum potassium $\geq 5 \mathrm{mEq} / \mathrm{L}$. Avoidance of an aldosterone antagonist in a patient who met all guideline criteria for therapy was also defined as inconsistent with the guidelines (Figure 2). Readmission rate within 30 days was defined as any readmission on days 0 to 30 post discharge and within 90 days as any readmission within days 0 to 90 . If more than $1 \mathrm{EF}$ was measured during the hospital stay, the following method determined which EF was utilized (in order from most to least preferred): multigated acquisition (MUGA) scan, transesophageal echocardiogram, transthoracic echocardiogram, pharmacologic stress test, and cardiac catheterization. One missing value for estimated GFR was imputed as 


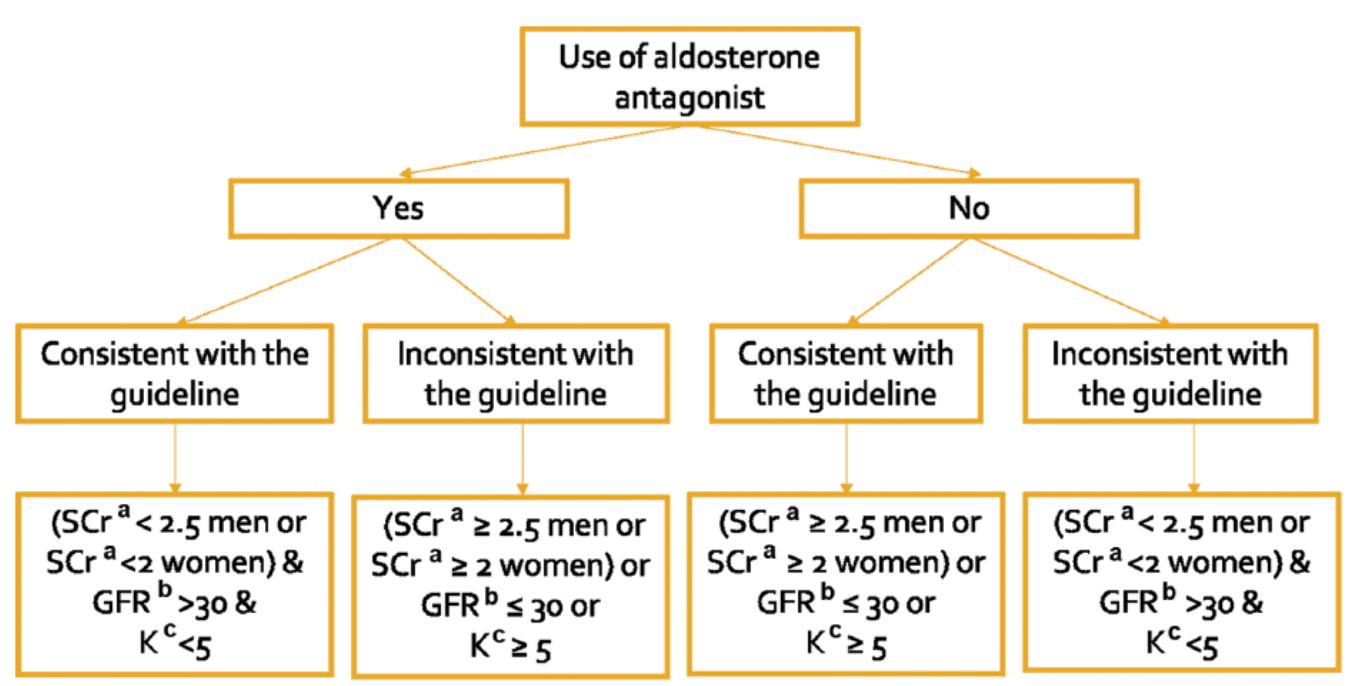

Figure 2. Definitions for appropriate use of aldosterone antagonists. Note. $\mathrm{SCr}=$ serum creatinine; GFR = glomerular filtration rate.

${ }^{\mathrm{a}} \mathrm{SCr}$ in units $\mathrm{mg} / \mathrm{dL}$.

${ }^{\mathrm{b}} \mathrm{GFR}$ in units $\mathrm{mL} / \mathrm{min}$.

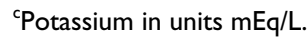

$\leq 30 \mathrm{~mL} / \mathrm{min}$ for 1 patient, who was 84 years old with a serum creatinine of $1.5 \mathrm{mg} / \mathrm{dL}$.

\section{Statistical Methods}

Patient characteristics were summarized using descriptive statistics, including frequencies and proportions, means and standard deviations, and medians and interquartile ranges. Comparisons between 30- and 90-day readmission rates of those receiving and not receiving an aldosterone antagonist were made using Pearson's 2-sided chi-square test.

\section{Results}

Between August 16, 2011, and June 5, 2013, a total of 848 hospitalizations with a primary discharge diagnosis of HF were screened and 346 patients met inclusion for the study. Demographic data are shown in Table 1. Of note, $87.6 \%$ of our patients were African American. Only 13 patients (3.8\%) had a discharge potassium level greater than $5 \mathrm{mEq} / \mathrm{L}$. The median serum creatinine was $1.4 \mathrm{mg} / \mathrm{dL}$ with an interquartile range of 0.7 and $1.1 \mathrm{mg} / \mathrm{dL}$ and with an interquartile range of 0.7 in males $(n=202)$ and females $(n=144)$, respectively; 47 patients (13.7\%) had estimated GFRs less than $30 \mathrm{~mL} / \mathrm{min}$. Sixty-two percent of patients $(n=216)$ were discharged on standard therapy of ACE inhibitor or ARB combined with a beta-blocker (Table 1).

One hundred twenty-one patients (35\%) received an aldosterone antagonist at discharge. Of these patients, 116 received an aldosterone antagonist consistent with the
Table I. Baseline Patient Data.

\begin{tabular}{lc}
\hline $\mathrm{N}=346$ & $\mathrm{n}(\%)$ \\
\hline Age, $\mathrm{y}$ & $62.6(24-95)$ \\
Sex & \\
Male & $202(58.4)$ \\
Female & $146(41.6)$ \\
Ethnicity & \\
African American & $303(87.6)$ \\
Caucasian & $40(11.6)$ \\
Other & $3(0.9)$ \\
Serum potassium $>5$ mEq/L & \\
eGFR (mL/min) & $13(3.8)$ \\
$\geq 30$ & \\
$<30$ & $298(86.1)$ \\
Other therapies utilized & $48(13.9)$ \\
Loop diuretic & \\
ACEl & $295(85.3)$ \\
ARB & $227(65.6)$ \\
Beta-blocker & $30(8.7)$ \\
Nitrate & $287(83.0)$ \\
Hydralazine & $83(24)$ \\
Digoxin & $72(20.8)$ \\
Pertinent medical history & $171(49.4)$ \\
Diabetes mellitus & \\
Coronary artery disease & $163(47.1)$ \\
\hline
\end{tabular}

Note. $\mathrm{ACEI}=$ angiotensin-converting enzyme inhibitor; $\mathrm{ARB}=$ angiotensin receptor blocker; eGFR = estimated glomerular filtration rate.

${ }^{a}$ All potassium values were checked but only abnormal values were recorded. 
Table 2. Aldosterone Antagonist Use ( $N=346)$.

\begin{tabular}{lclc}
\hline $\begin{array}{l}\text { Received an aldosterone } \\
\text { antagonist, } \mathrm{n}(\%)\end{array}$ & $121(35.0)$ & $\begin{array}{c}\text { Did not receive an } \\
\text { aldosterone antagonist, } \mathrm{n}(\%)\end{array}$ & $225(65.2)$ \\
\hline Consistent with guidelines & $116(95.9)$ & Consistent with guidelines & $55(24.4)$ \\
Inconsistent with guidelines & $5(4.1)$ & Hyperkalemia & $6(2.7)$ \\
Hyperkalemia & $3(2.4)$ & Renal Dysfunction & $45(20.0)$ \\
Renal dysfunction & $2(1.7)$ & Both & $4(1.8)$ \\
Both & $0(0)$ & Inconsistent with guidelines & $170(75.6)$ \\
\hline
\end{tabular}

Table 3. Readmission Rates.

\begin{tabular}{lccr}
\hline Outcomes & $\begin{array}{c}\text { Received an aldosterone } \\
\text { antagonist }(\mathrm{n}=\mid 21)\end{array}$ & $\begin{array}{c}\text { Did not receive an aldosterone } \\
\text { antagonist }(\mathrm{n}=225)\end{array}$ & Total \\
\hline $0-30$ days* $^{*}$ & $17(27.9)$ & $44(72.1)$ & $61(17.6)$ \\
$0-90$ days $^{\dagger}$ & $32(29.6)$ & $76(70.4)$ & $108(31.3)$ \\
\hline
\end{tabular}

$* P=.204$, Pearson's chi-square test.

${ }^{\dagger} P=.179$, Pearson's chi-square test.

guidelines. Therapy was considered inconsistent with the guidelines in 3 patients with hyperkalemia and 2 patients with renal dysfunction (Table 2). The remaining $65 \%$ of patients were not provided an aldosterone antagonist at discharge, yet only 55 of these 225 patients actually had at least 1 contraindication to therapy. Of these patients, 6 had hyperkalemia alone, 4 had hyperkalemia and renal dysfunction, and 45 patients had renal dysfunction. In addition, of those not receiving an aldosterone antagonist $(\mathrm{n}=225), 170$ $(75.6 \%)$ were eligible for aldosterone antagonist therapy with no contraindication present.

Sixty-one patients $(17.6 \%)$ were readmitted to the academic medical center within 30 days. Seventeen $(27.9 \%)$ of these patients received an aldosterone antagonist at discharge compared with $44(72.1 \%)$ who did not receive an aldosterone antagonist $(P=.24)$. Readmission within 90 days occurred for 108 patients (31.2\%), with 32 patients (29.6\%) in the aldosterone therapy group compared with 76 patients $(70.4 \%)$ in the group who did not receive an aldosterone antagonist $(P=.19)$ (Table 3$)$. Of the 5 patients who received an aldosterone antagonist and had a contraindication to therapy, 2 patients were readmitted. One patient had hyperkalemia (30 days readmit), and the other patient had renal failure (readmit 90 days). Of the 170 patients, who did not receive an aldosterone antagonist without contraindication to therapy, $31(18.2 \%)$ patients were readmitted within 30 days and $52(30.6 \%)$ were readmitted within 90 days.

\section{Discussion}

Despite the current ACCF/AHA guideline recommendations and recent literature, this study found that only $35 \%$ patients received an aldosterone antagonist at discharge. Our study adds to the growing body of literature regarding underuse of aldosterone antagonists. It is our hope that this investigation will encourage hospital-based pharmacists and other health care professionals to be more vigilant regarding the use of this valuable class of agents for reduced EF patients. This is the first study that observed the use of aldosterone antagonists in a patient population that consisted of greater than $85 \%$ African Americans. Although there were previous concerns regarding hyperkalemia from the RALES study, only 5 (4.1\%) patients received an aldosterone antagonist when a contraindication existed. It is important to note that only $62 \%$ of HF patients were discharged on standard therapy of beta-blocker and ACE inhibitor or ARB, and this finding could reflect why there was underutilization of aldosterone antagonists in this study. Patients may not have been on standard therapy due to hypotension or other contraindications to therapy; however, this study did not observe these findings.

We note that during the time period of our study, the 2009 Guidelines ${ }^{8}$ applied; with the 2013 Guidelines, ${ }^{5}$ the following statement is included: Aldosterone receptor antagonists are recommended to reduce morbidity and mortality following an acute myocardial infarction in patients who have a left ventricular ejection fraction (LVEF) of $40 \%$ or less who develop symptoms of HF or who have a history of diabetes mellitus, unless contraindicated. Subsequent studies examining aldosterone antagonist use should address these comorbidities. $^{9}$

In a retrospective study, Chamsi-Pasha et $\mathrm{al}^{10}$ found that aldosterone antagonists continue to be underutilized in patients admitted with acute decompensated HF. The most frequent reason for in-hospital discontinuation of aldosterone antagonists was increased serum creatinine and worsening of kidney function. The authors pointed out the importance of careful follow-up after aldosterone antagonist therapy initiation. Rossignol et $\mathrm{al}^{11}$ reported that in patients with HF receiving optimal treatment, worsening renal function and hyperkalemia were more common after 
eplerenone was initiated. However, the occurrence of these adverse effects did not eliminate the survival benefit of aldosterone antagonist therapy. This study further reiterates the importance of utilizing appropriate therapy as contraindications were minimal as compared with other studies. Inampudi et $\mathrm{a}^{12}$ reported that spironolactone therapy was not associated with a reduction in HF readmission and all-cause mortality. In hospitalized patients with reduced EF and advanced chronic kidney disease, spironolactone use was associated with higher all-cause readmission, but no association was found with all-cause mortality or HF readmission. This study found more frequent readmissions in patients at 30 and 90 days who did not receive an aldosterone antagonist at discharge compared with those who received aldosterone antagonist, although not statistically significant likely due to population size. The use of aldosterone antagonist may provide a small beneficial effect as well as cost-benefit. Interestingly, patients who had a contraindication to therapy had increased readmission rates compared with those without contraindication. Despite many factors that could contribute to readmission, it could be suggested that patients with reduced EF regardless of pharmacotherapy would be at increased risk of readmission.

Simopoulos et $\mathrm{al}^{13}$ recently reported that eplerenone decreased the incidence of postoperative atrial fibrillation in patients undergoing on-pump cardiac surgery, when added to standard HF treatment. The majority of patients in this study had $\mathrm{EF}<40 \%{ }^{13}$

Limitations to our investigation include the inherent problems with retrospective studies. In addition, this was a singlecenter study, and a larger patient population may have shown a difference in 30- and 90-day readmission rates between groups.

Current and past literature reiterates the proven benefits of aldosterone antagonists in certain patient populations, especially those with reduced EF HF. Despite this evidence, these agents remain underutilized. ${ }^{6,714-18}$ Finally, very recent evidence suggests reduced risk of hyperkalemia during the treatment of HF with aldosterone antagonists by use of sacubitril/valsartan versus enalapril. ${ }^{19}$

\section{Conclusions}

Aldosterone antagonists still remain underutilized despite current clinical guideline recommendations. Members of the health care team need to be more vigilant regarding appropriate use of aldosterone antagonists to help ensure optimal patient outcomes.

\section{Authors' Note}

At the time of this study, Dr. Patterson was a PGY2 Internal Medicine Pharmacy Resident at Methodist University Hospital, Memphis, and Dr. Ulrich was a Doctor of Pharmacy candidate at the University of Tennessee Health Science Center. Dr. Patterson is currently with Methodist South Hospital.

\section{Declaration of Conflicting Interests}

The author(s) declared no potential conflicts of interest with respect to the research, authorship, and/or publication of this article.

\section{Funding}

The author(s) received no financial support for the research, authorship, and/or publication of this article.

\section{References}

1. Pitt B, Zannad F, Remme WJ, et al; Randomized Aldactone Evaluation Study Investigators. The effect of spironolactone on morbidity and mortality in patients with severe heart failure. $N$ Engl J Med. 1999;341:709-717.

2. Pitt B, Remme W, Zannad F, et al. Eplerenone, a selective aldosterone blocker, in patients with left ventricular dysfunction after myocardial infarction. N Engl J Med. 2003;348(14):13091321.

3. Zannad F, McMurray JJ, Krum H, et al. Eplerenone in patients with systolic heart failure and mild symptoms. $N$ Engl J Med. 2011;364(1):11-21.

4. Pitt B, Pfeffer MA, Assmann SF, et al. Spironolactone for heart failure with preserved ejection fraction. $N$ Engl J Med. 2014;370(15):1383-1392.

5. Yancy CW, Jessup M, Bozkurt B, et al. ACCF/AHA Guideline for the management of heart failure: a report of the American College of Cardiology Foundation/American Heart Association Task Force on Practice Guidelines. J Am Coll Cardiol. 2013;62:e147-e239.

6. Albert NM, Yancy CW, Liang L, et al. Use of aldosterone antagonists in heart failure. JAMA. 2009;302:1658-1665.

7. Curtis LH, Mi X, Qualls LG, et al. Transitional adherence and persistence in the use of aldosterone antagonist therapy in patients with heart failure. Am Heart J. 2013;165:979-986.

8. Hunt SA, Abraham WT, Chin MH, et al. Focused update incorporated into the ACC/AHA 2005 guidelines for the diagnosis and management of heart failure in adults: a report of the American College of Cardiology Foundation/American Heart Association Task Force on Practice Guidelines. Circulation. 2009;119:e391-e479.

9. Hamaguchi S, Kinugawa S, Tsuchihashi-Makaya M, et al. Spironolactone use at discharge was associated with improved survival in hospitalized patients with systolic heart failure. Am Heart J. 2010;160:1156-1162.

10. Chamsi-Pasha MA, Dupont M, Al Jaroudi WA, Tang WH. Utilization pattern of mineralocorticoid receptor antagonists in contemporary patients hospitalized with acute decompensated heart failure: a single-center experience. J Card Fail. 2014;20:229-235.

11. Rossignol P, Dobre D, McMurray JJ, et al. Incidence, determinants, and prognostic significance of hyperkalemia and worsening renal function in patients with heart failure receiving the mineralocorticoid receptor antagonist eplerenone or placebo in addition to optimal medical therapy: results from the Eplerenone in Mild Patients Hospitalization and Survival Study in Heart Failure (EMPHASIS-HF). Circ Heart Fail. 2014;7:51-58.

12. Inampudi C, Parvataneni S, Morgan CJ, et al. Spironolactone use and higher hospital readmission for Medicare beneficiaries 
with heart failure, left ventricular ejection fraction $<45 \%$, and estimated glomerular filtration rate $<45 \mathrm{ml} / \mathrm{min} / 1.73 \mathrm{~m}^{2} . \mathrm{Am} J$ Cardiol. 2014;114:79-82.

13. Simopoulos V, Tagarakis G, Hatziefthimiou A, et al. Effectiveness of aldosterone antagonists for preventing atrial fibrillation after cardiac surgery in patients with systolic heart failure: a retrospective study. Clin Res Cardiol. 2015;104:3137.

14. Bress AP, King JB, Brixner D, et al. Pharmacotherapy treatment patterns, outcomes, and health resources utilization among patients with heart failure with reduced ejection fraction at a U.S. academic medical center. Pharmacotherapy. 2016;36:174-186.

15. Vaduganathan M, Dei Cas A, Mentz RJ, et al. Mineralocorticoid receptor antagonist use in hopspitalized patients with heart failure, reduced ejection fraction, and diabetes mellitus (from the EVEREST Trial). Am J Cardiol. 2014;114:743-750.
16. Franco G, Biagio F, Battista ZG, et al. ALERT-HF: adherence to guidelines in the treatment of patients with chronic heart failure. J Cardiovasc Med. 2014;15:491-497.

17. Bohm M, Tschope C, Wirtz JH, et al. Treatment of heart failure in real-world clinical practice: findings from the REFLECT-HF registry in patients with NYHA class II symptoms and a reduced ejection fraction. Clin Cardiol. 2015;38:200-207.

18. Miller RJ, Howlett JG. Retrospective review of in hospital use of mineralocorticoid receptor antagonists for high risk patients following myocardial infarction. BMC Cardiovasc Disord. 2015;10:46.

19. Desai AS, Vardeny O, Claggett B, et al. Reduced risk of hyperkalemia during treatment of heart failure with mineralocorticoid receptor antagonists by use of sacubitril/valsartan compared with enalapril: a secondary analysis of the PARADIGM-HF trial. JAMA Cardiol. 2017;2:79-85. 